\title{
Effects of Polymorphisms in the Serotonin Transporter Promoter-Linked Polymorphic Region on Postthoracotomy Pain Severity
}

\author{
Aya Kimura $\mathbb{D}^{\prime}$ \\ Hiroyuki Yamasaki' \\ Haruka Ishii ${ }^{2}$ \\ Hisako Yoshida ${ }^{2}$ \\ Motoko Shimizu ${ }^{3}$ \\ Takashi Mori' \\ 'Department of Anesthesiology, Osaka \\ City University Graduate School of \\ Medicine, Osaka City, Osaka, Japan; \\ ${ }^{2}$ Department of Medical Statistics, Osaka \\ City University Graduate School of \\ Medicine, Osaka City, Osaka, Japan; \\ ${ }^{3}$ Department of Anesthesiology, \\ Sumitomo Hospital, Osaka City, Osaka, \\ Japan
}

Purpose: Serotonin (5-HT) is highly associated with pain modulation. The human 5-HT transporter (5-HTT) gene (SLC6A4) features several polymorphisms in its promoter region (5-HTTLPR) that affect the 5-HTT expression. The S allele of 5-HTTLPR induces low 5-HT tone, and it may influence the modulation of chronic pain. Meanwhile, pain occurs in $40-50 \%$ of patients after thoracic surgery, and its mechanism remains under investigation. This study assessed the role of 5-HTTLPR polymorphisms in postthoracotomy pain severity. Patients and Methods: A total of 178 patients undergoing pneumonectomy were enrolled. The genotypes of 5-HTTLPR were divided into two groups: S/S group and S/L or L/L group. Linear mixed-effects models were used to assess the association between 5-HTTLPR genotypes and the numerical rating scale (NRS) score change over time.

Results: Among the participants, data were obtained for 162 patients. The genotype distribution was as follows: S/S, 67.3\%; S/L or L/L, 32.7\%. No significant difference in patient characteristics was found between the genotype groups. There was no significant interaction between the 5-HTTLPR genotypes and the NRS score change over time ( $\mathrm{p}=$ 0.842).

Conclusion: Polymorphisms in 5-HTTLPR were not associated with postthoracotomy pain severity.

Keywords: 5-HTTLPR, pain modulation, numerical rating scale

\section{Introduction}

Persistent postsurgical pain is a significant problem for a large number of patients. It decreases patients' quality of life and increases their economic burden. Surgery is essential for serious diseases, but $10-80 \%$ of patients experience pain after various types of surgery. ${ }^{1,2}$ The consequences vary widely among patients, causing both physical and mental disabilities.

Thoracotomy has been described as a high-risk surgery that induces persistent postsurgical pain. ${ }^{3}$ Postthoracotomy pain syndrome (PTPS) has been defined as pain lasting for $>2$ months after surgery by the International Association for Study of Pain. ${ }^{4}$ A large number of studies have focused on the predictors of PTPS. ${ }^{5,6}$ The risk factors for PTPS are classified into preoperative, intraoperative, and postoperative factors. Neuropathic changes after surgery have been demonstrated to be associated with chronic pain. ${ }^{2,7}$ However, the underlying mechanism is highly complex. Preoperative conditions, especially genetic factors, have emerged as novel predictors of postoperative chronic pain. ${ }^{8,9}$
Correspondence: Aya Kimura Department of Anesthesiology, Osaka City University Graduate School of Medicine, 1-5-7 Asahimachi, Abeno-ku, Osaka City, Osaka, 545-8586, Japan

Tel +8I-6-6645-2I86

$\mathrm{Fax}+81-6-6645-2489$

Email ayal226kimura@gmail.com 
The perception of pain varies among individuals, as it is a subjective experience influenced by combinations of factors such as sex, races, emotions, sensations, culture, and genes. ${ }^{10-12}$ Genetic studies have therefore been conducted to demonstrate the mechanism of pain modulation in the central nervous system. Serotonin (5-hydroxytryptamine [5-HT]) is a neurotransmitter that is highly involved in the pain mechanism. 5-HT is associated with the modulation of nociceptive signals both through the peripheral and central nervous systems, and it regulates mood. ${ }^{13,14}$ The 5-HT transporter (5-HTT) is an essential regulator of 5-HT that transfers the neurotransmitter from synapses to presynaptic neurons and terminates extracellular signaling. 5-HTT regulates the concentration of 5-HT and manages postsynaptic signaling. It has been revealed that the effectiveness of opioids depends on the 5-HTT genotype. ${ }^{15} 5$-HTT in humans is encoded by SLC6A4, one of the most studied genes in the neurobiology field. The promoter region of the gene contains several polymorphisms, and this variation affects the expression of the 5-HTT gene. The polymorphic region consists of a 43-base pair insertion/deletion known as the 5-HTTlinked polymorphic region (5-HTTLPR). This insertion/ deletion results in two variants with different lengths and activities: the short allele (S) and long allele (L). The $\mathrm{S}$ allele is associated with reduced 5-HTT gene transcription and thus diminished 5-HTT expression. Previous studies illustrated that the S allele is relevant to various diseases and conditions such as depression, schizophrenia, autism, addiction, ${ }^{16}$ anxiety disorder, ${ }^{17}$ obsessive-compulsive disorder, ${ }^{18}$ and chronic pain including fibromyalgia. ${ }^{19}$ The prevalence of the $\mathrm{S} / \mathrm{S}$ genotype in Japanese populations is reported to be 59-62\%, compared with $23-35 \%$ for the S/L genotype and $1-7 \%$ for the $\mathrm{L} / \mathrm{L}$ genotype. ${ }^{20}$ Considering the distribution of the genotypes in Japanese populations, we divided participants into the $\mathrm{S} / \mathrm{S}$ group and the $\mathrm{S} / \mathrm{L}$ or $\mathrm{L} /$ L group.

The aim of this study was to investigate the relationship of the incidence and severity of PTPS with 5-HTT expression. We hypothesized that patients with low 5-HTT expression were more likely to suffer from severe PTPS. To the best of our knowledge, this is the first large multicenter study to assess the association between 5-HT-linked genes and the mechanism of PTPS.

\section{Materials and Methods}

\section{Participants}

The study protocol was approved by the Ethical Committee of Osaka City University Graduate School of Medicine (Date of approval: August 3, 2017, Number: 3792, Registry number: UMIN000030176) and the Ethical Committee of Sumitomo Hospital (Date of approval: September 15, 2017, Number: 29-12). Patients aged $\geq 20$ years with an American Society of Anesthesiologists physical status class of 1-4 who were scheduled to undergo pneumonectomy for lung cancer were recruited from Osaka City University Hospital and Sumitomo Hospital between 2017 and 2018, and followup data collection ended in 2019. The exclusion criteria were current psychological illness and cognitive deficits. A total of 178 patients were enrolled. This study was conducted in accordance with the general principles outlined in the Declaration of Helsinki. Written informed consent was obtained from all participants.

\section{Study Protocol}

The participants completed the Pain Catastrophizing Scale (PCS) and provided a genomic DNA sample from the oral mucosa before surgery. After surgery, the pain numerical rating scale (NRS) and questionnaires about the interventions for pain relief were completed after 1 day and 3, 6, and 12 months of follow-up. The follow-up interview was conducted via a letter or an e-mail.

\section{Anesthesia and Surgery}

Open thoracic surgery or video-assisted thoracic surgery was selected by surgeons in accordance with their basic practice. For postoperative analgesia, thoracic epidural catheter was inserted at the T4-T6 level before surgery, and $1.5 \mathrm{~mL}$ of $1 \%$ lidocaine plus adrenaline were administered to test the procedure. Then, $0.25 \%$ levobupivacaine was continuously administered at $2-5 \mathrm{~mL} / \mathrm{h}$ according to the severity of pain and blood pressure until postoperative day 2 or 3 . For patients receiving anticoagulation therapy and hemodialysis therapy and those with spinal disease, epidural anesthesia was not administered. For alternative pain management, intrathoracic intercostal block with $0.33 \%$ ropivacaine was performed at the end of the surgery, and intravenous patientcontrolled analgesia with fentanyl at $20 \mu \mathrm{g} / \mathrm{h}$ was given for 2 or 3 days after surgery in patients without epidural anesthesia. 


\section{PCS}

The PCS is used to assess the psychological distress of and dysfunctional adjustment to pain. ${ }^{21,22}$ The PCS is a selfadministered questionnaire consisting of 13 items. Each item is rated from 0 to 4 to measure the frequency of painrelated thoughts and feelings, with 0 meaning "not at all" and 4 meaning "always." The sum of the 13 items comprises the PCS score, which ranges from 0 to 52 . Participants were instructed to reflect on thoughts or feelings during past painful experiences.

\section{Assessment of Pain Scaling}

The NRS is recognized as the gold standard direct assessment for pain. The scale is scored from 0 to 10 , with 0 indicating no pain and 10 indicating the worst possible pain. The NRS score was measured 1 day and 3, 6, and 12 months after thoracic surgery. Participants were asked to rate their average pain on the first postoperative day. After discharge, patients were mailed questionnaires, and they reported their average pain status in the last 48 hours.

\section{Genotyping}

DNA was extracted from patients' oral mucosa using QIAamp DNA Mini Kit (Qiagen, Dusseldorf, Germany) according to the manufacturer's instructions and analyzed via polymerase chain reaction (PCR). The forward and reverse primer sequences for 5-HTT polymorphisms were 5'-GCAACCTCCCAGCAACTCCCTGTA-3' and 5'GAGGTGCAGGGGGATGCTGGAA-3', respectively. Each sample was amplified using KOD FX Neo (TOYOBO, Osaka, Japan). The $20-\mu \mathrm{L}$ PCR reaction mixture contained $2 \times$ buffer $(10 \mu \mathrm{L})$, deoxyribonucleotide triphosphates $(4 \mu \mathrm{L})$, forward and reverse primers (each 0.6 $\mu \mathrm{L})$, KOD FX neo DNA polymerase $(0.4 \mu \mathrm{L})$, distilled water $(0.4 \mu \mathrm{L})$, and $25-50 \mathrm{ng}$ of genomic DNA $(2 \mu \mathrm{L})$. The cycling program was as follows: $94^{\circ} \mathrm{C}$ for $2 \mathrm{~min}, 35$ cycles of $98^{\circ} \mathrm{C}$ for $10 \mathrm{~s}$ and $64^{\circ} \mathrm{C}$ for $20 \mathrm{~s}$, and storage at $4^{\circ}$ C. Then, PCR production was analyzed via electrophoresis using a 3130xl Genetic Analyzer (Thermo Fisher Scientific, Waltham, MA, USA). The two allele variants were identified on the basis of the PCR fragment size (S allele, $138 \mathrm{bp}$; $\mathrm{L}$ allele, $182 \mathrm{bp}$ ). Samples were classified into three genotypes: $\mathrm{S} / \mathrm{S}, \mathrm{S} / \mathrm{L}$, and $\mathrm{L} / \mathrm{L}$.

\section{Statistical Analysis}

The primary outcome of the present study was the interaction between pain severity and 5-HTTLPR genotypes.
To assess the relationship between 5-HTTLPR polymorphisms and the NRS score change over time, statistical analysis was performed using a linear mixed-effects model. The linear mixed-effects model contained the fixed effects of time, 5-HTTLPR genotypes, and the interaction of time and genotypes and the random effect of interception correlated to each subject, recognized as a cluster. Patients were grouped by genotype into the $\mathrm{S} / \mathrm{S}$ group or $\mathrm{S} / \mathrm{L}$ or $\mathrm{L} / \mathrm{L}$ group. The adjustment variables included preoperative persistent pain, age, sex, PCS, and analgesia after surgery (with vs without epidural anesthesia). To confirm the interactions of genotype and time with variables including preoperative persistent pain, operation types, sex, PCS, and analgesia after surgery, we conducted a similar mixed-effect model analysis in the entire population.

The sample size was calculated to detect a $1.5 \times$ mean difference in the NRS score at 1 year after surgery. Assuming a sample size ratio of 2.3:1 between the $\mathrm{S} / \mathrm{S}$ group and the $\mathrm{S} / \mathrm{L}$ or $\mathrm{L} / \mathrm{L}$ group and an $\mathrm{SD}$ of 2.5 , power analysis indicated that a sample size of 150 patients was sufficient to ensure $90 \%$ power with an alpha of 0.05 , allowing an attrition rate of $10 \%$. The Kruskal-Wallis test and chi-squared test were used to compare continuous and categorical variables, respectively, between the genotype groups. The level of significance was set at $\mathrm{p}<0.05$. All statistical analyses were performed using $\mathrm{R}$ (version 3.6.1 with $\mathrm{R}$ packages).

\section{Results}

Data were obtained for 162 patients. The genotype distribution was as follows: $\mathrm{S} / \mathrm{S}, 67.3 \%$ and $\mathrm{S} / \mathrm{L}$ or $\mathrm{L} / \mathrm{L}, 32.7 \%$. As presented in Table 1, age, sex, presence of chronic pain, existence of epidural anesthesia, total intravenous anesthesia, surgical type (VATS or open thoracotomy), and preoperative pain catastrophizing scale were similar among $\mathrm{S} / \mathrm{S}$, and $\mathrm{S} / \mathrm{L}$ or $\mathrm{L} / \mathrm{L}$ groups.

\section{Effects of Genotypes on NRS}

After adjustment, NRS decreased significantly as time progressed until one year after surgery. No significant difference was found in the NRS score changes according to the 5-HTTLPR genotype $(p=0.928)$. The mixed-effect model results revealed no significant interaction between the genotype groups and the NRS score change over time (5-HTTLPR genotype $\times$ elapsed time, $p=0.842$, Figure 1$)$. 
Table I Patients' Characteristics by 5-HTTLPR Genotype

\begin{tabular}{|c|c|c|c|c|c|}
\hline & $\mathbf{S} / \mathbf{S}$ & S/L & L/L & Total & $p$ value \\
\hline $\mathrm{n}$ & 109 & 49 & 4 & 162 & \\
\hline Age $(y r)$ & $71.0(65.0-76.0)$ & $70.0(65.0-75.0)$ & $69.5(65.3-75.0)$ & $70.5(65.0-76.0)$ & 0.95 \\
\hline Male & $71.6 \%$ & $65.3 \%$ & $100 \%$ & $70.4 \%$ & 0.307 \\
\hline Chronic pain & $15.6 \%$ & $18.4 \%$ & $0 \%$ & $26 \%$ & 0.614 \\
\hline Epidural anesthesia & $86.2 \%$ & $87.8 \%$ & $100 \%$ & $87.0 \%$ & 0.712 \\
\hline TIVA & $1.8 \%$ & $2.0 \%$ & $0 \%$ & $1.9 \%$ & 0.958 \\
\hline VATS & $92.7 \%$ & $87.8 \%$ & $100 \%$ & $91.4 \%$ & 0.492 \\
\hline PCS & $18.0(\mid 1.0-28.0)$ & $22.0(12.0-32.0)$ & $15(4.5-27.5)$ & $20.0(11.0-29.0)$ & 0.503 \\
\hline
\end{tabular}

Notes: Continuous variables present as mean (interquartile range), categorical variables present as frequency; Significance is defined as $p<0.05$

Abbreviations: TIVA, total intravenous anesthesia; VATS, video-assisted thoracic surgery; PCS, pain catastrophizing scale.

Effects of the Interactions Among Time, Genotypes, and Patient Variables on NRS

The mixed-effect model results demonstrated significant interactions among time, genotypes, and epidural anesthesia (5-HTTLPR genotypes $\times$ epidural anesthesia $\times$ elapsed time, $\mathrm{p}=0.05)$. Differences in the NRS score change over time between the $\mathrm{S} / \mathrm{S}$ group and the $\mathrm{S} / \mathrm{L}$ or $\mathrm{L} / \mathrm{L}$ group were associated with epidural anesthesia (Figure 2). It indicated the effect on NRS elapsed change was different depending on the interaction of the genotypes and epidural anesthesia. Conversely, there were no significant differences in the inter-

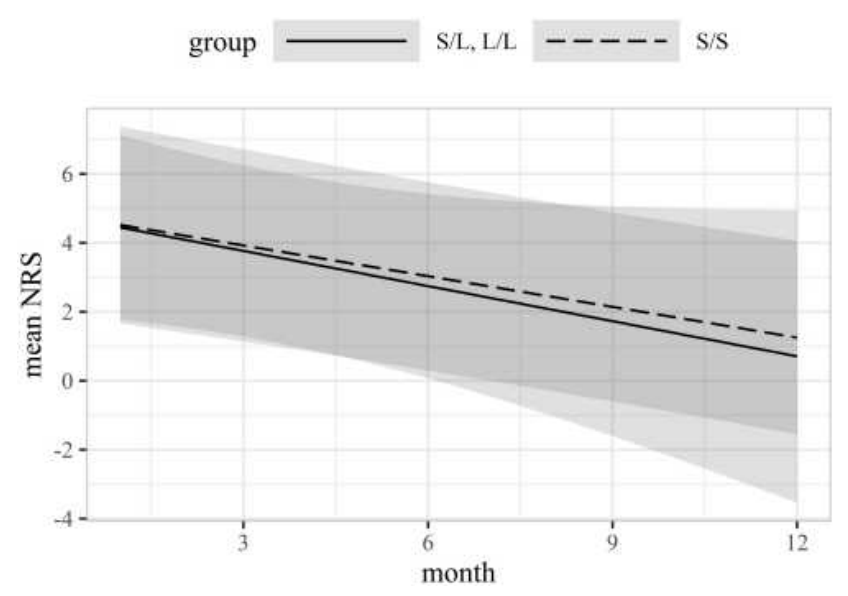

Figure I Effect of serotonin transporter promoter-linked polymorphic region genotypes on NRS score changes using a linear-mixed effect model. The shaded area indicates the $95 \%$ confidence interval.

Abbreviation: NRS, numerical rating scale. action among time, genotypes, and patient variables including preoperative chronic pain, operative types, PCS, and sex (5-HTTLPR genotypes $\times$ preoperative chronic pain $\times$ elapsed time, $\mathrm{p}=0.96$; 5-HTTLPR genotypes $\times$ operation types $\times$ elapsed time, $\mathrm{p}=0.627$; 5-HTTLPR genotypes $\times$ PCS $\times$ elapsed time, $p=0.305 ; 5$-HTTLPR genotypes $\times$ sex $\times$ elapsed time, $p=0.938$; Figures $3,4,5$, and 6).

\section{Assessment of Pain Scaling}

Table 2 indicates the NRS scores measured 1 day and 3,6, and 12 months after thoracic surgery. There was no group

A

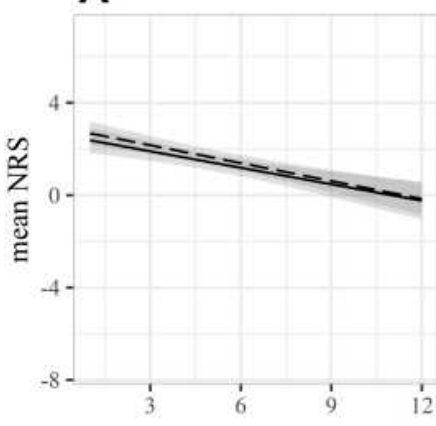

S/L, L/L - - - S/S

B

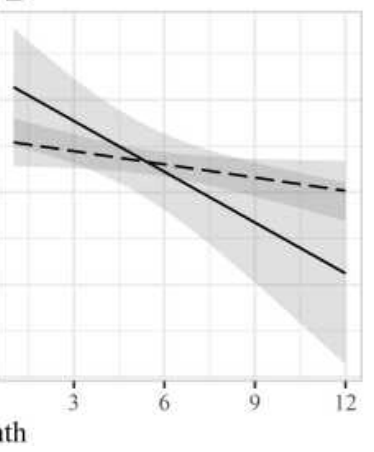

Figure 2 Effect of the interaction between serotonin transporter promoter-linked polymorphic region genotypes and epidural anesthesia on NRS score changes using a linear-mixed effect model. (A) NRS score changes in patients who received epidural anesthesia. (B) NRS score changes in patients who did not receive epidural anesthesia. The shaded area indicates the $95 \%$ confidence interval. Abbreviation: NRS, numerical rating scale. 


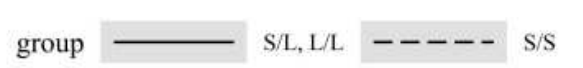

A

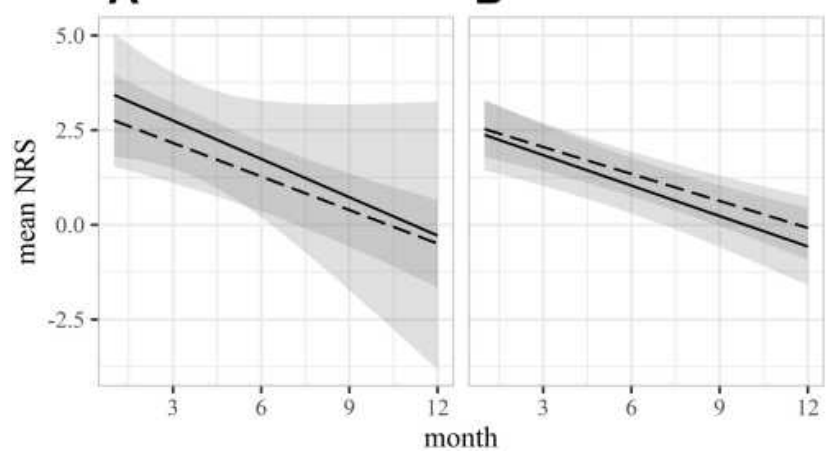

Figure 3 Effect of the interaction between serotonin transporter promoter-linked polymorphic region genotypes and preoperative persistent pain on NRS score changes using a linear-mixed effect model. (A) NRS score changes in patients with preoperative persistent pain. (B) NRS score changes in patients without preoperative persistent pain. The shaded area indicates the $95 \%$ confidence interval. Abbreviation: NRS, numerical rating scale.

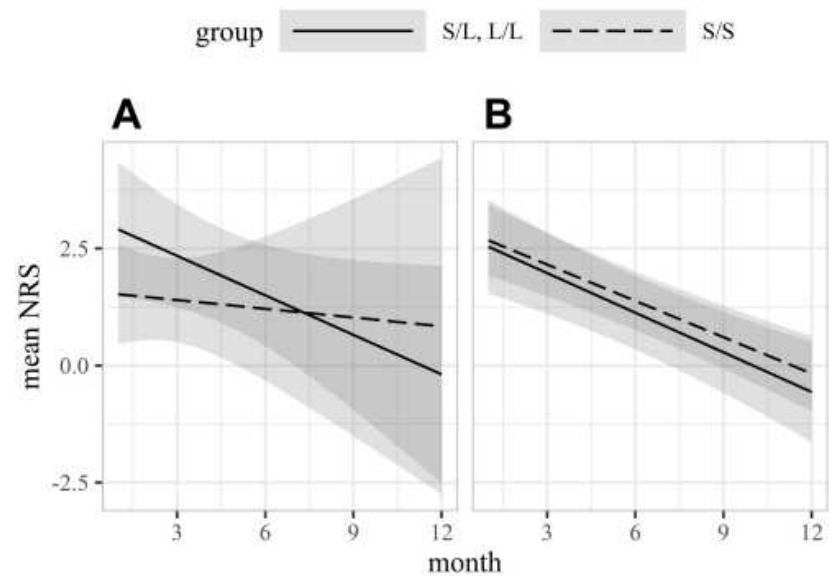

Figure 4 Effect of the interaction between serotonin transporter promoter-linked polymorphic region genotypes and surgery types on NRS score changes using a linear-mixed effect model. (A) NRS score changes in patients who underwent open thoracic surgery. (B) NRS score changes in patients who underwent videoassisted thoracic surgery. The shaded area indicates the $95 \%$ confidence interval. Abbreviation: NRS, numerical rating scale.

significant difference in the values of NRS between the two groups.

\section{Discussion}

The main finding of the current study was that 5-HTTLPR polymorphisms alone did not influence postthoracotomy pain severity. Our results were consistent with previous findings of no association between 5-HTTLPR polymorphisms and pain perception in patients with chronic pain. ${ }^{21}$ Our data also indicated an interaction among time, genotypes, and epidural anesthesia with changes in the NRS

group $\longrightarrow$ S/L, L/L $----\mathrm{S} / \mathrm{S}$

A B

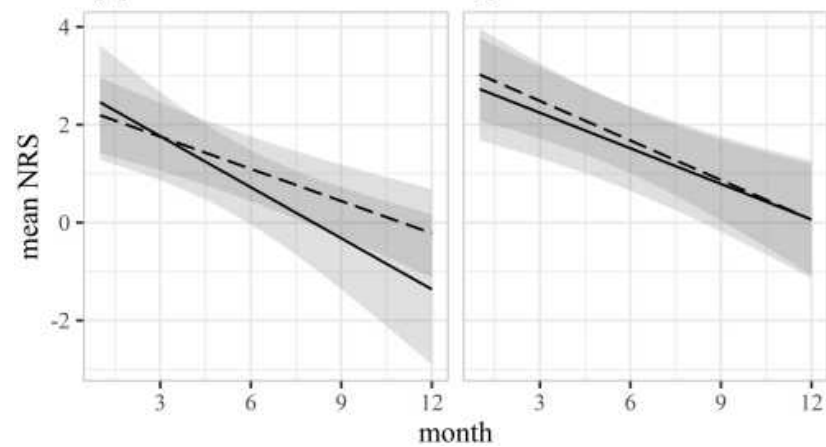

Figure 5 Effect of the interaction between serotonin transporter promoter-linked polymorphic region genotypes and the preoperative Pain Catastrophizing Scale (PCS) on NRS score changes using a linear-mixed effect model. (A) NRS score changes in patients with a preoperative PCS below the mean. (B) NRS score changes in patients with a preoperative PCS above the mean. The shaded area indicates the $95 \%$ confidence interval.

Abbreviation: NRS, numerical rating scale.

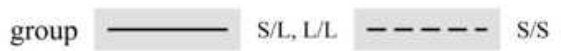

A

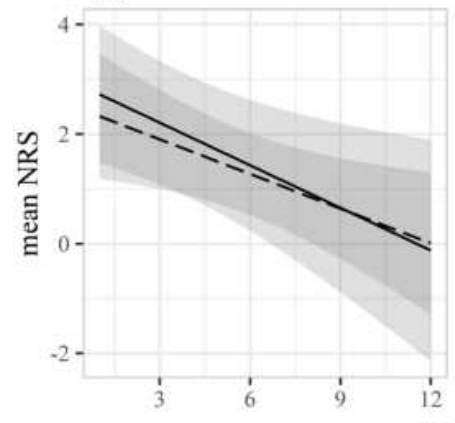

month
B

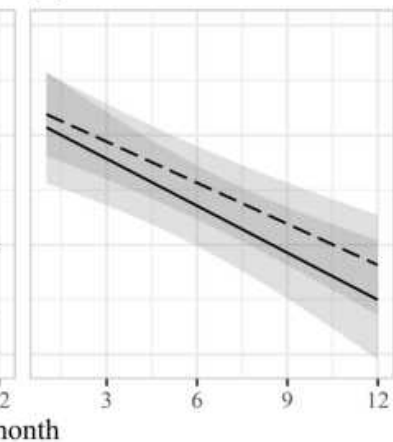

Figure 6 Effect of the interaction between serotonin transporter promoter-linked polymorphic region genotypes and sex on NRS score changes using a linear-mixed effect model. (A) NRS score changes in male patients. (B) NRS score changes in female patients. The shaded area indicates the $95 \%$ confidence interval.

Abbreviation: NRS, numerical rating scale.

score over time. This suggested that epidural anesthesia may contribute to changes over time in postthoracotomy pain severity depending on the patient's serotonergic tone.

There has been great concern about persistent pain after surgery. PTPS is a severe form of chronic pain associated with surgery. Despite a large number of studies, ${ }^{7,23-25}$ the mechanism of PTPS remains unclear. Considering the subjective nature of pain perception, the interindividual differences are associated with genetic variations. 5-HT is a major neurotransmitter that is notably involved in pain perception. It is well known that 5-HTT 
Table 2 NRS Values After Surgery

\begin{tabular}{|l|c|c|c|c|}
\hline Time After Surgery & I Day & 3 Months & 6 Months & I2 Months \\
\hline NRS (S/S) & 4.52 & 3.92 & 3.03 & 1.25 \\
\hline NRS (S/L or L/L) & 4.44 & 3.77 & 2.75 & 0.71 \\
\hline Difference & $\begin{array}{c}0.08 \\
(-1.27 \text { to I.42) }\end{array}$ & $\begin{array}{c}0.16 \\
(-0.84 \text { to I.15) }\end{array}$ & $\begin{array}{c}0.29 \\
(-1.30 \text { to I.87) }\end{array}$ & $\begin{array}{c}0.54 \\
(-3.39 \text { to } 4.47)\end{array}$ \\
\hline
\end{tabular}

Notes: Values of NRS present as mean; Difference of NRS between S/S vs S/L or L/L was present as mean and $95 \%$ confidence interval.

Abbreviation: NRS, numerical rating scale.

plays an important role in 5-HT regulation, and genetic variations in SLC6A4 influence 5-HT reuptake. ${ }^{26}$ 5-HTTLPR is located in the SLC6A4 promoter, and it has allelic variations, the most common of which are the $\mathrm{S}$ and $\mathrm{L}$ alleles. We hypothesized that the variations of 5-HTTLPR affect the incidence of chronic pain after thoracic surgery via the pain control pathways associated with 5-HT and mood changes following postsurgical pain. Previous studies indicated that the genotypes of 5-HTT differ among the races. The prevalence of the $\mathrm{S}$ allele is $79 \%$ in Asian populations versus $42 \%$ in Western populations. ${ }^{26}$ The $\mathrm{L}$ allele is expressed more frequently in Caucasians than in Asians. The genotypes of 5-HTTLPR are mainly classified into S/S, S/L, and L/L. The prevalence of the $\mathrm{S} / \mathrm{S}$ genotype is higher in Asians $(60 \%)$ than in Caucasians $(22 \%)$; contrarily, $1-13 \%$ of Asians and $29-43 \%$ of Caucasians carry the L/L genotype. ${ }^{26}$ The $\mathrm{S}$ allele is known to be associated with low 5-HTT expression, which is linked with low 5-HT tone. A number of studies reported the relevance of the $\mathrm{S}$ allele with chronic pain and depressive symptoms. ${ }^{16-19}$ Previous studies demonstrated the association of 5-HTTLPR polymorphisms with the perception of pain in Caucasian populations. ${ }^{27,28}$ To our knowledge, there are fewer reports about the relationship between pain modulation and genotypes in Asian populations than in Caucasian populations. In this study, we enrolled Japanese patients and examined the relevance of 5-HTTLPR polymorphisms to the mechanism of PTPS. Our result revealed no significant difference in the NRS score between the genotype groups. This suggested that the 5-HTT genotypes alone had no association with the modulation of PTPS. The A/G SNP in 5-HTTLPR differs between the $\mathrm{S}$ and $\mathrm{L}$ alleles. The $\mathrm{G}$ allele, which is minor to the A allele, almost always exists with the $\mathrm{L}$ allele, and it decreases the expression of the $\mathrm{S}$ allele. This difference explains the functional effect on 5-HTTLPR. In addition to 5-HTT, the 5-HT system contains a large number of 5-HT receptors. These receptors consist of seven groups, and among them, the 5-HT1A receptor is most widely expressed. ${ }^{29}$ Therefore, the 5-HT1A receptor also plays an important role in the regulation of the central 5-HT tone. Concerning 5-HTTLPR polymorphism, the 5-HT1A receptor gene has variants that contribute to the expression of the 5-HT1A receptor. $^{30}$ This polymorphism influences central 5-HT regulation, which may have interactions with 5-HTT expression. Similar to the 5-HT signaling process, opioid signaling is also associated with chronic pain modulation. Genetic studies of the mu-opioid receptor (OPRM1) revealed a polymorphism in the OPRM1 gene, which controls the activation of the mu-opioid receptor. A previous study indicated that polymorphisms of OPRM1, 5-HTT, and 5-HT1A did not affect pain mechanism on their own, but there were genetic interactions between the mu-opioid receptor and 5-HT structures in the modulation of fibromyalgia. ${ }^{31}$ Considering these findings, genetic variation alone did not regulate the pain mechanism, but genetic interactions might influence pain regulation.

The PCS is widely used to predict chronic pain intensity by assessing the maladaptive response to pain. A large number of studies reported a significant association between PCS and pain-related outcomes including pain severity, response to pain treatment, and opioid prescription. ${ }^{22,32}$ High PCS scores are associated with severe pain experiences and chronic pain. ${ }^{33}$ Our result revealed no significant interaction of 5-HTTLPR polymorphisms and PCS scores with the changes in the NRS score over time, whereas previous research indicated that genotype variations in 5-HT3B were associated with the PCS score. ${ }^{34} 5$-HT is notably involved in the mechanism of pain perception, and 
further investigation about the relationship between the genotypes relevant to 5-HT and PCS scores may be encouraged.

Previous studies on 5-HTTLPR have shown that there is a significant association between sex and polymorphism in the regulation of serotonin tone for patients with chronic tension-type facial pain. ${ }^{35,36}$ However, our results indicated there was no significant interaction between genotypes and sex in the change of NRS score $(p=0.938)$. The mechanism of postoperative chronic pain modulation may be different compared with the other types of chronic pain including tension-type facial pain.

In the present study, the interaction of epidural anesthesia with 5-HTT genotypes was found to describe the difference in the trajectories of the NRS score $(p=0.05)$. A large number of studies about therapeutic interventions after thoracic surgery have been conducted. Epidural anesthesia has been considered a gold standard for pain management after thoracic surgery. ${ }^{37}$ A systematic review indicated that regional anesthesia reduced both acute and chronic pain after thoracotomy. ${ }^{3}$ Conversely, another study found that epidural anesthesia did not significantly prevent PTPS compared with systemic analgesia. ${ }^{38}$ Our result illustrated that epidural anesthesia appeared to provide pain relief for individuals carrying the $\mathrm{S} / \mathrm{S}$ genotype, considering the changes in the NRS score over time. Contrarily, the other genotypes were not associated with sensitivity to epidural anesthesia regarding the prevention of PTPS. The controversial effect of epidural anesthesia on the incidence of PTPS can be explained by these genetic variations of 5-HTT. However, in the current study, only a few subjects received non-epidural anesthesia pain management. Further studies are needed to elucidate the interaction among epidural anesthesia, 5-HTT, and PTPS.

Our study had several strengths including multicenter nature, and long evaluation period. In the current study, we performed analysis using a mixed-effect model to increase power and avoid biasing results compared with a simple linear regression model. Despite these advantages, some limitations must be addressed. First, this study only evaluated the association of 5-HTTLPR polymorphisms with the mechanism of PTPS. However, many factors contribute to pain modulation, such as the 5-HT receptor, catechol$O$-methyltransferase, and opioid receptors. These genes are known to have genotype variations similar to 5 -HTT, ${ }^{29,30,39}$ and we did not exclude the possible interactions of other pain-related genes. Second, given the incidence of 5-HTTLPR polymorphisms in Asian populations, we divided genotypes into the $\mathrm{S} / \mathrm{S}$ group and the $\mathrm{S} / \mathrm{L}$ or $\mathrm{L} / \mathrm{L}$ group, whereas previous studies grouped patients differently. ${ }^{28,40}$ This classification of the genotypes might have affected the findings. Third, $70 \%$ of the participants were male. This factor might restrict the ability of this study to demonstrate differences between the allelic groups because of the possible sex difference in serotonin metabolism. In addition, we only considered the $\mathrm{S}$ and $\mathrm{L}$ alleles. The existence of the A and $\mathrm{G}$ alleles influences individuals' 5-HT tone, which might affect the modulation of PTPS. Furthermore, we did not investigate the effects of epigenetic changes on the regulation of 5-HTT. Variations including DNA methylation might affect the result.

\section{Conclusion}

There was no association between the 5-HTTLPR polymorphism and PTPS over 1 year. Although 5-HTT plays an important role in pain modulation, the chronic pain mechanism might involve complicated gene interactions opposed to a single gene. Further genotyping studies are required to elucidate the potential association between 5-HT and pain modulation.

\section{Clinical Trial Number}

UMIN000030176

\section{Author Contributions}

All authors made a significant contribution to the work reported, whether that is in the conception, study design, execution, acquisition of data, analysis and interpretation, or in all these areas; took part in drafting, revising or critically reviewing the article; gave final approval of the version to be published; have agreed on the journal to which the article has been submitted; and agree to be accountable for all aspects of the work.

\section{Funding}

This study was supported by JSPS KAKENHI Grant Number 17K16750.

\section{Disclosure}

The authors report no conflicts of interest in this work.

\section{References}

1. Kehlet H, Jensen TS, Woolf CJ. Persistent postsurgical pain: risk factors and prevention. Lancet. 2006;367:1618-1625. doi:10.1016/ S0140-6736(06)68700-X 
2. Borsook D, Kussman BD, George E, Becerra LR, Burke DW. Surgically induced neuropathic pain: understanding the perioperative process. Ann Surg. 2013;257:403-412. doi:10.1097/ SLA.0b013e3182701a7b

3. Humble SR, Dalton AJ, Li L. A systematic review of therapeutic interventions to reduce acute and chronic post-surgical pain after amputation, thoracotomy or mastectomy. Eur $J$ Pain. 2015;19:451-465. doi:10.1002/ejp.567

4. Schug SA, Pogatzki-Zahn EM. Chronic pain after surgery or injury. Pain Clin Updates. 2011;19:1-4.

5. Grosen K, Laue Petersen G, Pfeiffer-Jensen M, Hoejsgaard A, Pilegaard HK. Persistent post-surgical pain following anterior thoracotomy for lung cancer: a cross-sectional study of prevalence, characteristics and interference with functioning. Eur J Cardiothorac Surg. 2013;43:95-103. doi:10.1093/ejcts/ezs159

6. Bayman EO, Parekh KR, Keech J, Selte A, Brennan TJ. A prospective study of chronic pain after thoracic surgery. Anesthesiology. 2017;126:938-951. doi:10.1097/ALN.0000000000001576

7. Dualé C, Guastella V, Morand D, et al. Characteristics of the neuropathy induced by thoracotomy: a 4-month follow-up study with psychophysical examination. Clin J Pain. 2011;27:471-480. doi:10.1097/AJP.0b013e31820e12d4

8. Matre D, Olsen MB, Jacobsen LM, Klein T, Gjerstad J. Induction of the perceptual correlate of human long-term potentiation (LTP) is associated with the 5-HTT genotype. Brain Res. 2013;1491:54-59. doi:10.1016/j.brainres.2012.10.045

9. Olsen MB, Jacobsen LM, Schistad EI, et al. Pain intensity the first year after lumbar disc herniation is associated with the A118G polymorphism in the opioid receptor mu 1 gene: evidence of a sex and genotype interaction. $J$ Neurosci. 2015;19:451-465.

10. Leknes S, Tracey I. A common neurobiology for pain and pleasure. Nat Rev Neurosci. 2008;9:314-320. doi:10.1038/nrn2333

11. Rhudy JL, Williams AE, McCabe KM, Russell JL, Maynard LJ. Emotional control of nociceptive reactions $(\mathrm{ECON})$ : do affective valence and arousal play a role? Pain. 2008;136:250-261. doi:10.1016/j.pain.2007.06.031

12. Coghill RC. Individual differences in the subjective experience of pain: new insights into mechanisms and models. Headache. 2010;50:1531-1535. doi:10.1111/j.1526-4610.2010.01763.x

13. Benarroch EE. Descending monoaminergic pain modulation: bidirectional control and clinical relevance. Neurology. 2008;71:217-221. doi:10.1212/01.wnl.0000318225.51122.63

14. Lowry CA, Lightman SL, Nutt DJ. That warm fuzzy feeling: brain serotonergic neurons and the regulation of emotion. J Psychopharmacol. 2009;23:392-400. doi:10.1177/0269881108099956

15. Kosek E, Jensen KB, Lonsdorf TB, Schalling M, Ingvar M. Genetic variation in the serotonin transporter gene (5-HTTLPR, rs25531) influences the analgesic response to the short acting opioid Remifentanil in humans. Mol Pain. 2009;5:37. doi:10.1186/1744-8069-5-37

16. Gelernter J. SLC6A4 polymorphism, population genetics, and psychiatric traits. Hum Genet. 2014;133:459-461. doi:10.1007/s00439-013$1412-2$

17. You JS, Hu SY, Chen B, Zhang HG. Serotonin transporter and tryptophan hydroxylase gene polymorphisms in Chinese patients with generalized anxiety disorder. Psychiatr Genet. 2005;15:7-11. doi:10.1097/00041444-200503000-00002

18. Hu XZ, Lipsky RH, Zhu G, et al. Serotonin transporter promoter gain-of-function genotypes are linked to obsessive-compulsive disorder. Am J Hum Genet. 2006;78:815-826. doi:10.1086/503850

19. D'Agnelli S, Arendt-Nielsen L, Gerra MC, et al. Fibromyalgia: genetics and epigenetics insights may provide the basis for the development of diagnostic biomarkers. Mol Pain. 2019;15:1744806918819944. doi:10.1177/1744806918819944
20. Goldman N, Glei DA, Lin YH, Weinstein M. The serotonin transporter polymorphism (5-HTTLPR): allelic variation and links with depressive symptoms. Depress Anxiety. 2010;27:260-269. doi:10.1002/da.20660

21. Potvin S, Larouche A, Normand E, et al. No relationship between the ins del polymorphism of the serotonin transporter promoter and pain perception in fibromyalgia patients and healthy controls. Eur J Pain. 2010;14:742-746. doi:10.1016/j.ejpain.2009.12.004

22. Sharifzadeh Y, Kao MC, Sturgeon JA, Rico TJ, Mackey S, Darnall BD. Pain catastrophizing moderates relationships between pain intensity and opioid prescription: nonlinear sex differences revealed using a learning health system. Anesthesiology. 2017;127:136-146. doi:10.1097/ALN.0000000000001656

23. Doan LV, Augustus J, Androphy R, Schechter D, Gharibo C. Mitigating the impact of acute and chronic post-thoracotomy pain. J Cardiothorac Vasc Anesth. 2014;28:1048-1056. doi:10.1053/j. jvca.2014.02.021

24. Guastella V, Mick G, Soriano C, et al. A prospective study of neuropathic pain induced by thoracotomy: incidence, clinical description, and diagnosis. Pain. 2011;152:74-81. doi:10.1016/j. pain.2010.09.004

25. Maguire MF, Latter JA, Mahajan R, Beggs FD, Duffy JP. A study exploring the role of intercostal nerve damage in chronic pain after thoracic surgery. Eur J Cardiothorac Surg. 2006;29:873-879. doi:10.1016/j.ejcts.2006.03.031

26. Iurescia S, Seripa D, Rinaldi M. Role of the 5-HTTLPR and SNP promoter polymorphisms on serotonin transporter gene expression: a closer look at genetic architecture and in vitro functional studies of common and uncommon allelic variants. Mol Neurobiol. 2016;53:5510-5526. doi:10.1007/s12035-015-9409-6

27. Lindstedt F, Berrebi J, Greayer E, et al. Conditioned pain modulation is associated with common polymorphisms in the serotonin transporter gene. PLoS One. 2011;6:e18252. doi:10.1371/journal. pone. 0018252

28. Lindstedt F, Lonsdorf TB, Schalling M, Kosek E, Ingvar M. Perception of thermal pain and the thermal grill illusion is associated with polymorphisms in the serotonin transporter gene. PLoS One. 2011;6:e17752. doi:10.1371/journal.pone.0017752

29. Nichols DE, Nichols CD. Serotonin receptors. Chem Rev. 2008;108:1614-1641. doi:10.1021/cr078224o

30. Lindstedt F, Karshikoff B, Schalling M, et al. Serotonin-1A receptor polymorphism (rs6295) associated with thermal pain perception. PLoS One. 2012;7:e43221. doi:10.1371/journal.pone.0043221

31. Tour J, Lofgren M, Mannerkorpi K, et al. Gene-to-gene interactions regulate endogenous pain modulation in fibromyalgia patients and healthy controls-antagonistic effects between opioid and serotonin-related genes. Pain. 2017;158:1194-1203. doi:10.1097/j. pain.0000000000000896

32. Ziadni MS, Sturgeon JA, Darnall BD. The relationship between negative metacognitive thoughts, pain catastrophizing and adjustment to chronic pain. Eur J Pain. 2018;22:756-762. doi:10.1002/ejp.1160

33. Khan RS, Ahmed K, Blakeway E, et al. Catastrophizing: a predictive factor for postoperative pain. Am J Surg. 2011;201:122-131. doi:10.1016/j.amjsurg.2010.02.007

34. Horjales-Araujo E, Demontis D, Lund EK, et al. Polymorphism in serotonin receptor $3 \mathrm{~B}$ is associated with pain catastrophizing. PLoS One. 2013;8:e78889. doi:10.1371/journal.pone.0078889

35. Agius AM, Jones NS, Muscat R. Serial blood serotonin levels in a randomized controlled trial comparing the efficacy of low-dose amitriptyline, amitriptyline with pindolol and surrogate placebo in patients with chronic tension-type facial pain. Rhinology. 2013;51:236-242. doi:10.4193/Rhino13.019

36. Agius AM, Muscat R: Chronic tension-type facial pain - a pilot study on HTTLPR genetic polymorphisms. 2019. 
37. Joshi GP, Bonnet F, Shah R, et al. A systematic review of randomized trials evaluating regional techniques for posthoracotomy analgesia. Anesth Analg. 2008;107:1026-1040. doi:10.1213/01. ane.0000333274.63501.ff

38. Kampe S, Lohmer J, Weinreich G, Hahn M, Stamatis G, Welter S. Epidural analgesia is not superior to systemic postoperative analgesia with regard to preventing chronic or neuropathic pain after thoracotomy. J Cardiothorac Surg. 2013;8:127. doi:10.1186/17498090-8-127
39. Diatchenko L, Nackley AG, Slade GD, et al. CatecholO-methyltransferase gene polymorphisms are associated with multiple pain-evoking stimuli. Pain. 2006;125:216-224. doi:10.1016/j. pain.2006.05.024

40. Kunz M, Hennig J, Karmann AJ, Lautenbacher S. Relationship of 5-HTTLPR polymorphism with various factors of pain processing: subjective experience, motor responsiveness and catastrophizing. PLoS One. 2016;11:e0153089. doi:10.1371/journal.pone.0153089

\section{Publish your work in this journal}

The Journal of Pain Research is an international, peer reviewed, open access, online journal that welcomes laboratory and clinical findings in the fields of pain research and the prevention and management of pain. Original research, reviews, symposium reports, hypothesis formation and commentaries are all considered for publication. The manuscript

Submit your manuscript here: https://www.dovepress.com/journal-of-pain-research-journa management system is completely online and includes a very quick and fair peer-review system, which is all easy to use. Visit http:// www.dovepress.com/testimonials.php to read real quotes from published authors. 